\title{
Vertically nested LES for high-resolution simulation of the surface layer in PALM (version 5.0)
}

\author{
Sadiq Huq ${ }^{1}$, Frederik De Roo ${ }^{1,4}$, Siegfried Raasch ${ }^{3}$, and Matthias Mauder ${ }^{1,2}$ \\ ${ }^{1}$ Institute of Meteorology and Climate Research, Atmospheric Environmental Research (IMK-IFU), Karlsruhe Institute \\ of Technology (KIT), Kreuzeckbahnstrasse 19, 82467 Garmisch-Partenkirchen, Germany \\ ${ }^{2}$ Institute of Geography and Geoecology (IfGG), Karlsruhe Institute of Technology, Kaiserstrasse 12, \\ 76131 Karlsruhe, Germany \\ ${ }^{3}$ Institute of Meteorology and Climatology, Leibniz Universität Hannover, Hanover, Germany \\ ${ }^{4}$ Norwegian Meteorological Institute, Oslo, Norway
}

Correspondence: Matthias Mauder (matthias.mauder@kit.edu)

Received: 15 November 2018 - Discussion started: 3 December 2018

Revised: 16 April 2019 - Accepted: 4 June 2019 - Published: 28 June 2019

\begin{abstract}
Large-eddy simulation (LES) has become a wellestablished tool in the atmospheric boundary layer research community to study turbulence. It allows three-dimensional realizations of the turbulent fields, which large-scale models and most experimental studies cannot yield. To resolve the largest eddies in the mixed layer, a moderate grid resolution in the range of 10 to $100 \mathrm{~m}$ is often sufficient, and these simulations can be run on a computing cluster with a few hundred processors or even on a workstation for simple configurations. The desired resolution is usually limited by the computational resources. However, to compare with tower measurements of turbulence and exchange fluxes in the surface layer, a much higher resolution is required. In spite of the growth in computational power, a high-resolution LES of the surface layer is often not feasible: to fully resolve the energy-containing eddies near the surface, a grid spacing of $\mathrm{O}(1 \mathrm{~m})$ is required. One way to tackle this problem is to employ a vertical grid nesting technique, in which the surface is simulated at the necessary fine grid resolution, and it is coupled with a standard, coarse, LES that resolves the turbulence in the whole boundary layer. We modified the LES model PALM (Parallelized Large-eddy simulation Model) and implemented a two-way nesting technique, with coupling in both directions between the coarse and the fine grid. The coupling algorithm has to ensure correct boundary conditions for the fine grid. Our nesting algorithm is realized by modifying the standard third-order Runge-Kutta time stepping to allow communication of data between the two grids. The
\end{abstract}

two grids are concurrently advanced in time while ensuring that the sum of resolved and sub-grid-scale kinetic energy is conserved. We design a validation test and show that the temporally averaged profiles from the fine grid agree well compared to the reference simulation with high resolution in the entire domain. The overall performance and scalability of the nesting algorithm is found to be satisfactory. Our nesting results in more than $80 \%$ savings in computational power for 5 times higher resolution in each direction in the surface layer.

\section{Introduction}

Turbulence in the atmospheric boundary layer (ABL) encompasses a wide range of scales from the boundary-layer scale down to the viscous dissipation scale. In ABL flows, Reynolds numbers $(R e)$ of $10^{8}$ are commonly encountered. Explicit simulation of the Navier-Stokes equations down to the dissipative scales (DNS: direct numerical simulation) for atmospheric processes is prohibitively expensive, as the required number of grid points in one direction scales with $R e^{3 / 4}$ (Reynolds, 1990). This corresponds to a threedimensional ABL simulation domain with a total number of grid points of order $10^{17}$. The supercomputers of today cannot fit more than $10^{12}$ grid points in their memory. To be able to compute turbulence processes in the atmosphere nevertheless, the concept of large-eddy simulation (LES) was introduced already a few decades ago, 
e.g., Deardorff (1974), Moeng and Wyngaard (1988) and Schmidt and Schumann (1989), where the presence of a sub-grid-scheme allows that only the most energetic eddies are resolved. One of the first large-eddy simulations (LESs) by Deardorff (1974) used 64000 grid points to simulate a domain of $5 \mathrm{~km} \times 5 \mathrm{~km} \times 2 \mathrm{~km}$ with a grid resolution of $(125,125,50) \mathrm{m}$. The size of one such grid cell is just sufficient to resolve the dominant large eddies, and there are just enough grid points to represent the ABL. As computing power progressed, higher resolution and larger domains became possible. By the time of Schmidt and Schumann (1989) the number of grid cells had risen to $160 \times 160 \times 48$, simulating an ABL of $8 \mathrm{~km} \times 8 \mathrm{~km} \times 2.4 \mathrm{~km}$ with a resolution of $(50,50,50) \mathrm{m}$. Khanna and Brasseur (1998) used $128^{3}$ grid points to simulate a domain of $3 \mathrm{~km} \times 3 \mathrm{~km} \times 1 \mathrm{~km}$ to study buoyancy and shear-induced local structures of the ABL. Patton et al. (2016) used $(2048,2048,1024)$ grid points with a grid resolution of $(2.5,2.5,2) \mathrm{m}$ to study the influence of atmospheric stability on canopy turbulence. More recently, Kröniger et al. (2018) used $13 \times 10^{9}$ grid points to simulate a domain of $30.72 \mathrm{~km} \times 15.36 \mathrm{~km} \times 2.56 \mathrm{~km}$ to study the influence of stability on the surface-atmosphere exchange and the role of secondary circulations in the energy exchange. The atmospheric boundary layer community has greatly benefited from the higher spatial resolution available in these LES to study turbulent processes that cannot be obtained in field measurements. Still, especially in heterogeneous terrain, near topographic elements and buildings or close to the surface, the required higher resolution is not always attainable due to computational constraints. In spite of the radical increase in the available computing power over the last decade, largeeddy simulation of high Reynolds number atmospheric flows with very high resolution in the surface layer remains a challenge. Considering the size of the domain required to reproduce boundary-layer-scale structures, it is computationally demanding to generate a single fixed grid that could resolve all relevant scales satisfactorily. Alternatively, local grid refinement is possible in the finite volume codes that are not restricted to structured grids. Flores et al. (2013) developed a solver for the OpenFOAM modeling framework to simulate atmospheric flows over complex geometries using an unstructured mesh approach. The potential of the adaptive mesh refinement technique in which the tree-based Cartesian grid is refined or coarsened dynamically, based on the flow structures, is demonstrated by van Hooft et al. (2018). In the finite difference models, a grid nesting technique can be employed to achieve the required resolution. In the nested grid approach, a parent domain with a coarser resolution simulates the entire domain, while a nested grid with a higher resolution extends only up to the region of interest. Horizontal nesting has been applied to several mesoscale models (Skamarock et al., 2008; Debreu et al., 2012). Horizontally nested LES-within-LES or LES embedded within a mesoscale simulation is available in the Weather Research and Forecast model (Moeng et al., 2007). Comparable grid nesting tech- niques are also widely employed by the engineering turbulence research community but often use different terminology. Nesting in codes with Cartesian grids is referred to as local or zonal grid algorithm (Kravchenko et al., 1996; Boersma et al., 1997; Manhart, 2004) and as overset mesh (Nakahashi et al., 2000; Kato et al., 2003; Wang et al., 2014) in unstructured or moving grid codes.

For our purposes, we will focus on vertical nesting; i.e., we consider a fine grid nested domain (FG) near the lower boundary of the domain and a coarse grid parent domain (CG) in the entirety of the boundary layer. While the latter's resolution $(<50 \mathrm{~m})$ is sufficient to study processes in the outer region where the dominant eddies are large and inertial effects dominate, such coarse resolution is not sufficient where fine-scale turbulence in the surface layer region is concerned. The higher resolution achieved by the vertical nesting will then allow a more accurate representation of the turbulence in the surface layer region, by resolving its dominant eddies. For studies that require very high resolution near the surface (e.g., virtual tower measurements, wakes behind obstacles, dispersion within street canyons for large cities), a nesting approach is an attractive solution due to the reduced memory requirement. A challenge of the vertically nested simulation is that the FG upper boundary conditions need to be correctly prescribed by the CG. Though vertical nesting is less common than the horizontal nesting, it has been implemented in some LES models. A non-parallelized vertical nesting was explored by Sullivan et al. (1996), but the code is not in the public domain, and we could not find any record of further development or application of this code in publications. A LES-within-LES vertical nesting is implemented by Zhou et al. (2018) in the Advanced Regional Prediction System (ARPS) model. We would like to point out that the vertical nesting available in the Weather Research and Forecast model (Daniels et al., 2016) is not a conventional vertical nesting because the parent and the child grid still have the same vertical extent; the child grid is only more refined in the vertical.

An analysis of different nesting procedures for mesoscale simulation was performed by Clark and Hall (1991); they coined the terms "one-way" and "two-way" interactions. In one-way interaction, only the FG receives information from the CG, and there is no feedback to the CG. In two-way interaction, the FG top boundary conditions are interpolated from the $C G$, and the $C G$ values in the overlapping region are updated with the FG resolved fields. The "update" process, referred to as "anterpolation" by Sullivan et al. (1996), is similar to the restriction operation in multi-grid methods. Harris and Durran (2010) used a linear 1-D shallow-water equation to study the influence of the nesting method on the solution and found the two-way interaction to be superior if the waves are well resolved. They introduce a filtered sponge boundary condition to reduce the amplitude of the reflected wave at the nested grid boundary. We will make use of the interpolation and anterpolation formulas of Clark and Farley (1984). Clark 
and Hall (1991) studied two different approaches for updating the CG values, namely "post-insertion" and "pressure defect correction". The two approaches were also investigated by Sullivan et al. (1996) in their vertical nesting implementation. In the post-insertion technique, once the Poisson equation for pressure is solved in the FG, the resolved fields are then anterpolated to the CG. In the pressure defect correction approach, the pressure in the $\mathrm{CG}$ and FG are matched by adding a correction term to the CG momentum equations, and an anterpolation operation is not required. Though Sullivan et al. (1996) note the pressure defect correction approach to be more elegant, no significant difference in the results was reported. In the following sections we describe the technical realization and numerical aspects of the two-way nesting algorithm. In the LES model PALM, a validation simulation is set up, and the results of the nested and stand-alone simulations are compared. A second simulation is set up to evaluate the computational performance of the algorithm. The practical considerations and the limitations of the two-way nesting are then discussed.

\section{Methods}

\subsection{Description of the standard PALM}

The Parallelized Large-eddy simulation Model (PALM) is developed and maintained at the Leibniz University of Hanover (Raasch and Schröter, 2001; Maronga et al., 2015). We give a quick summary of the model here and highlight the aspects which will reappear when discussing our nesting modifications. PALM is a finite difference solver for the non-hydrostatic incompressible Navier-Stokes equations in the Boussinesq approximation. PALM solves for six prognostic equations: the three components of the velocity field $(u, v, w)$, potential temperature $(\theta)$, humidity $(q)$ and the subgrid-scale kinetic energy $(e)$. The sub-grid-scale (SGS) turbulence is modeled based on the method proposed by Deardorff (1980). The equations for the conservation of mass, energy and moisture (Eqs. 1, 2, 3 and 4) are filtered over a grid volume on a Cartesian grid. Adopting the convention of Maronga et al. (2015), the overbar denoting the filtered variables is omitted. However, the overbar is shown for SGS fluxes. The SGS variables are denoted by a double prime. The prognostic equations for the resolved variables are

$$
\begin{aligned}
\frac{\partial u_{i}}{\partial t}= & -\frac{\partial u_{i} u_{j}}{\partial x_{j}}-\varepsilon_{i j k} f_{j} u_{k}+\varepsilon_{i 3 k} f_{3} u_{k_{\mathrm{g}, j}}-\frac{1}{\rho_{0}} \frac{\partial \pi^{*}}{\partial x_{i}} \\
& +g \frac{\theta_{\mathrm{v}}-\left\langle\theta_{\mathrm{v}}\right\rangle}{\theta_{\mathrm{v}}} \delta_{i 3}-\frac{\partial}{\partial x_{j}}\left(\overline{u_{i}^{\prime \prime} u_{j}^{\prime \prime}}-\frac{2}{3} e \delta_{i j}\right) \\
\frac{\partial u_{j}}{\partial x_{j}}= & 0, \\
\frac{\partial \theta}{\partial t}= & -\frac{\partial u_{j} \theta}{\partial x_{j}}-\frac{\partial}{\partial x_{k}}\left(\overline{u_{j}^{\prime \prime} \theta^{\prime \prime}}\right)-\frac{L_{\mathrm{V}}}{c_{p} \Pi} \Psi_{q_{\mathrm{v}}},
\end{aligned}
$$

$\frac{\partial q_{\mathrm{v}}}{\partial t}=-\frac{\partial u_{j} q_{\mathrm{v}}}{\partial x_{j}}-\frac{\partial}{\partial x_{k}}\left(\overline{u_{j}^{\prime \prime} q_{\mathrm{v}}^{\prime \prime}}\right)+\Psi_{q_{\mathrm{v}}}$.

The symbols used in the above equations are listed in Table 1. The 1.5-order closure parameterization modified by Moeng and Wyngaard (1988) and Saiki et al. (2000) assumes a gradient diffusion parameterization (Eqs. 6, 7, 8). The prognostic equation for the SGS turbulent kinetic energy (TKE) reads as

$\frac{\partial e}{\partial t}=-u_{j} \frac{\partial e}{\partial x_{j}}-\left(\overline{u_{i}^{\prime \prime} u_{j}^{\prime \prime}}\right) \frac{\partial u_{i}}{\partial x_{k}}+\frac{g}{\theta_{\mathrm{v}, 0}} \overline{u_{3}^{\prime \prime} \theta_{\mathrm{v}}^{\prime \prime}}-2 K_{\mathrm{m}} \frac{\partial e}{\partial x_{j}}-\epsilon$,

with the SGS fluxes modeled as

$\overline{u_{i}^{\prime \prime} u_{j}^{\prime \prime}}-\frac{2}{3} e \delta_{i j}=-K_{\mathrm{m}}\left(\frac{\partial u_{i}}{\partial x_{j}}+\frac{\partial u_{j}}{\partial x_{i}}\right)$,
$\overline{u_{i}^{\prime \prime} \theta^{\prime \prime}}=-K_{\mathrm{h}} \frac{\partial \theta}{\partial x_{i}}$

and

$\overline{u_{i}^{\prime \prime} q_{v}^{\prime \prime}}=-K_{\mathrm{h}} \frac{\partial \theta}{\partial x_{i}}$.

The eddy diffusivities are proportional to $e^{3 / 2}$ under convective conditions (Maronga et al., 2015). For a thorough description of the governing equations and parameterizations, see Maronga et al. (2015).

The prognostic equations are discretized on a staggered Arakawa $\mathrm{C}$ grid, where the scalars are evaluated in the center of the grid volume, and velocities are evaluated at the center of the faces of the grid volume in their respective direction. The advection terms are evaluated either with fifthorder upwind discretization according to Wicker and Skamarock (2002) or with a second-order scheme according to Piacsek and Williams (1970). The prognostic equations are integrated in time using a third-order Runge-Kutta (RK3) scheme. The low storage RK3 scheme with three sub-steps proposed by Williamson (1980) guarantees a stable numerical solution. The Poisson equation for pressure is solved with a fast Fourier transform (FFT) when periodic boundary conditions are applied in the lateral boundaries. There are three FFT algorithms available in PALM, with Fastest Fourier Transform in the West (FFTW) being the optimal method for large-scale simulations. Monin-Obukhov similarity theory (MOST) is assumed between the surface and the first grid point. A vertical zero pressure gradient at the surface guarantees the vertical velocity to be zero. Simulations can be driven by either prescribing the surface temperature or the surface sensible heat flux, similarly for the humidity. At the top of the simulation domain the horizontal velocities equal geostrophic wind, and the vertical velocity is set to zero. The pressure can assume either a Dirichlet condition of zero value or a Neumann condition of zero vertical gradient. The scalar values can have either a fixed value Dirichlet condition or a fixed gradient Neumann condition. The vertical gradient of SGS turbulent kinetic energy (TKE) is set to 
Table 1. List of symbols in the governing equations and parameterizations.

\begin{tabular}{ll}
\hline Symbol & Description \\
\hline$f_{i}$ & Coriolis parameter \\
$\rho_{0}$ & Density of dry air at the surface \\
$\pi^{*}$ & Modified perturbation pressure \\
$g$ & Gravitational acceleration \\
$\theta_{\mathrm{V}}$ & Virtual potential temperature \\
$L_{\mathrm{V}}$ & Latent heat of vaporization \\
$C_{p}$ & Heat capacity of dry air at constant pressure \\
$q_{v}$ & Specific humidity \\
$\Psi_{q_{v}}$ & Source/sink term of $q_{v}$ \\
$\Pi$ & Exner function for converting between temperature and potential temperature \\
$K_{\mathrm{h}}$ & SGS eddy diffusivity of heat \\
$K_{\mathrm{m}}$ & SGS eddy diffusivity of momentum \\
\hline
\end{tabular}

zero at both top and bottom boundaries. PALM is a parallelized model, and the standard way of parallelization is by dividing the three-dimensional domain into vertical columns, each of which is assigned to one processing element (PE). Each vertical column possesses a number of ghost points needed for computation of derivatives at the boundary of the sub-domains. Each PE can only access data for a single subdomain. All PEs execute the same program on a different set of data. For optimum load balancing between the PEs, the decomposed sub-domains should have the same size. In PALM, this condition is always satisfied as only sub-domains of the same size are allowed. The data exchange between PEs needed by the Poisson solver and to update the ghost points is performed via the Message Passing Interface (MPI) communication routines.

\subsection{Nested model structure}

\subsubsection{Fine grid and coarse grid configuration}

We are interested in achieving an increased resolution only in the surface layer, the lowest $10 \%$ of the boundary layer, where surface exchange processes occur and where eddies generated by surface heterogeneity and friction are smaller than the dominant eddies in the mixed layer. We set up the LES-within-LES case by maintaining the same horizontal extent for the FG and the CG to have the whole surface better resolved. We allow the vertical extent of the FG to be varied as needed, typically up to the surface layer height. This implementation of vertical grid nesting has two main challenges. The first challenge, that is purely technical in nature, is to implement routines that handle the communication of data between the CG and the FG. The second and the most important challenge is to ensure that the nesting algorithm yields an accurate solution in both grids. Below we use upper case symbols for fields and variables in the $\mathrm{CG}$ and lower case for the FG; e.g., $E$ and $e$ denote the sub-grid-scale turbulent kinetic energy (a prognostic variable in our LES) of CG and FG respectively. The nesting ratio is defined as the ratio of the CG spacing to the FG spacing, and $n_{x}=\Delta X / \Delta x$; corresponding symbols apply for $y$ and $z$ directions. The nesting ratios $n_{x}, n_{y}$ and $n_{z}$ have to be integers. It is possible to have either an odd or even nesting ratio, and it can be different in each direction. As the domain that is simulated in the FG is completely inside of the CG domain, each FG cell belongs to a $\mathrm{CG}$ cell. The two grids are positioned in such a way that a FG cell belongs to only one CG cell, and one CG cell is made up by a number of FG cells given by the product of the nesting ratios $n_{x} \times n_{y} \times n_{z}$. This means that if the grid nesting ratio is odd, there will be one FG cell whose center is exactly at the same position as the center of the coarse cell as shown in Fig. 1b. The collection of FG cells that correspond to one $\mathrm{CG}$ cell is denoted by $\mathcal{C}(I, J, K)$. The collection of $y z$ faces of the FG that corresponds to a $y z$ face of the $\mathrm{CG}$ is denoted by $\mathcal{C}_{x}\left(I_{\mathrm{s}}, J, K\right)$, where it is understood that the $I_{\mathrm{s}}$ index is an index on the staggered grid in the $x$ direction to denote the position of the face, and this is similar for the other types of faces. We have used $f_{x}=1 / n_{x}$ to denote the inverse of the nesting ratio in the $x$ dimension (corresponding symbols for $y$ and $z$ ). A schematic diagram of the overlapping grids is shown in Fig. 1a.

\subsubsection{Vertical nesting algorithm}

We implement a two-way interaction algorithm, shown in Fig. 2, because in our first trials we found that one-way nesting did not improve the FG representation satisfactorily and hence was not pursued further. The FG prognostic quantities are initialized by interpolating the $\mathrm{CG}$ values in the overlapping region. Optionally, the initialization of the FG can be delayed until the $\mathrm{CG}$ has reached a fully turbulent state. Both grids are restricted to have identical time steps. PALM finds the largest time step for each grid such that the CourantFriedrichs-Lewy (CFL) condition is individually satisfied, and the minimum of the two values is then chosen as the time integration step for both grids. The right-hand side of 


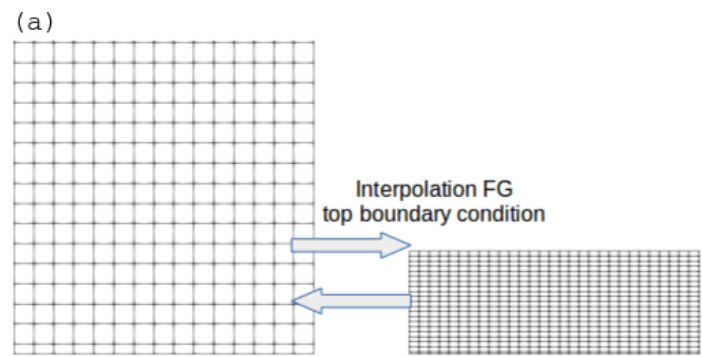

Anterpolation to the

overlapping region of the CG (b)

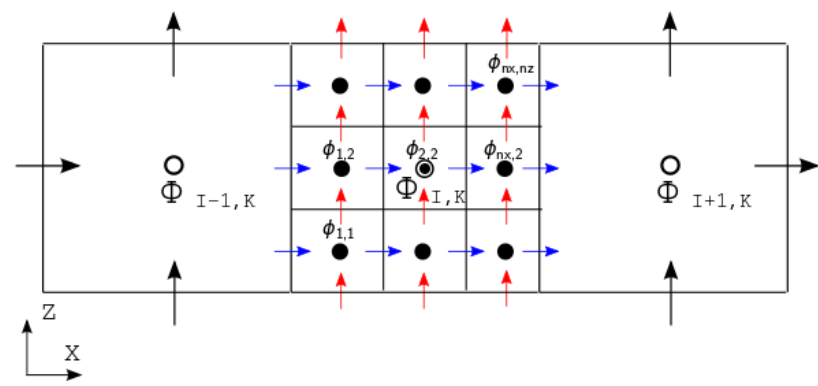

Figure 1. (a) Schematic of the interpolation and anterpolation between grids. The FG top boundary condition is interpolated from the CG. The CG prognostic quantities in the overlapping region are anterpolated from the FG. (b) Schematic of Arakawa C grid for two grids with nesting ratio of 3 . The black arrows and circles are CG velocity and pressure, respectively. The blue and red arrows are horizontal and vertical velocity, respectively, in the FG. The filled black circle is the FG pressure. The symbols $\Phi$ and $\phi$ represent CG and FG scalar quantities. $I$ and $K$ are CG indices and $n x$ and $n z$ are the nesting ratio in $x$ and $z$, respectively.

the prognostic equation except for the pressure is first computed concurrently in both grids. The values of $u, v, w, \theta$ and $q$ are then anterpolated to the $\mathrm{CG}$ in the overlapping region. The CG solves a Poisson equation for pressure. The new $u, v, w, \theta$ and $q$ fields in the CG are interpolated to set the FG Dirichlet top boundary conditions. The Poisson equation is then solved for pressure in the FG, and the vertical velocity in the FG is also updated by the pressure solver at this stage. Since all the velocity components follow a Dirichlet condition at the FG top boundary, only a Neumann condition is suitable for pressure (Manhart, 2004). PALM permits the use of a Neumann zero-gradient condition for pressure at both the top and bottom boundary. It is advisable to use a Neumann boundary condition at the top and the bottom for the CG too. The TKE is then anterpolated maintaining the Germano identity, and it is followed by the computation of SGS eddy diffusivity for heat $\left(k_{\mathrm{h}}\right)$ and momentum $\left(k_{\mathrm{m}}\right)$ in the CG. This procedure is repeated at every sub-step of the RungeKutta 3 time integration, and it ensures that the velocity field remains divergence free in both grids.

In the 1.5-order turbulence closure parameterization, all the sub-grid fluxes are derived from the turbulent kinetic energy and the resolved gradients at each time step. Therefore, the sub-grid fluxes do not have to be interpolated from CG to FG at the top boundary. Furthermore, in our implementation of the nesting method, we assume that most of the TKE is resolved well down to the inertial subrange, except for the lowest few grid layers. This allows us to use the zero-gradient Neumann boundary condition for TKE at the FG top boundary. We employ a simplified sponge layer by limiting the anterpolation of all prognostic quantities to one CG cell fewer than the nested height. This segregation of the anterpolation region in the $\mathrm{CG}$ and top boundary condition level of the FG ensures that the flow structures in the CG propagate into the FG without distortion due to numerical artifacts.

\subsection{Translation between grids}

\subsubsection{Interpolation}

For the boundary conditions at the top of the FG, the fields from the CG are interpolated to the FG, according to Clark and Farley (1984). We define the top of the FG as the boundary level just above the prognostic level of each quantity. In Eq. (10), $\Phi$ and $\phi$ represent CG and FG quantities, respectively. For the scalar fields, the interpolation is quadratic in all three directions. For the velocity components, the interpolation is linear in its own dimension and quadratic in the other two directions. The same interpolation formulation is also used to initialize all vertical levels of the fine grid domain at the beginning of the nested simulation. The interpolation is reversible as it satisfies the conservation condition of Kurihara et al. (1979):

$<\phi>=<\Phi>$.

For clarity, we illustrate the interpolation by focusing on one particular dimension, in this case $x$, but the same operation holds for $y$ and $z$. The interpolation in the $x$ dimension reads as

$\phi_{m}=\eta_{-}^{m} \Phi_{I-1}+\eta_{0}^{m} \Phi_{I}+\eta_{+}^{m} \Phi_{I+1}$,

with $m$ running from 1 to $n_{x}$, thus producing $n_{x}$ equations for each CG cell $I$. For the interpolation in $y$ and $z$ there will be two additional indices, producing $n_{x} \times n_{y} \times n_{z}$ equations for all the FG cells corresponding to the CG parent cell. For the quadratic interpolation a stencil with three legs is used, relating the prognostic value of a FG cell to the value of its parent $C G$ and the values of the immediate $C G$ neighbor on the left and on the right of the parent cell, e.g., $\Phi_{I-1}$ and $\Phi_{I+1}$ for the $x$ direction as shown in Fig. 1b. The stencil coefficients are 


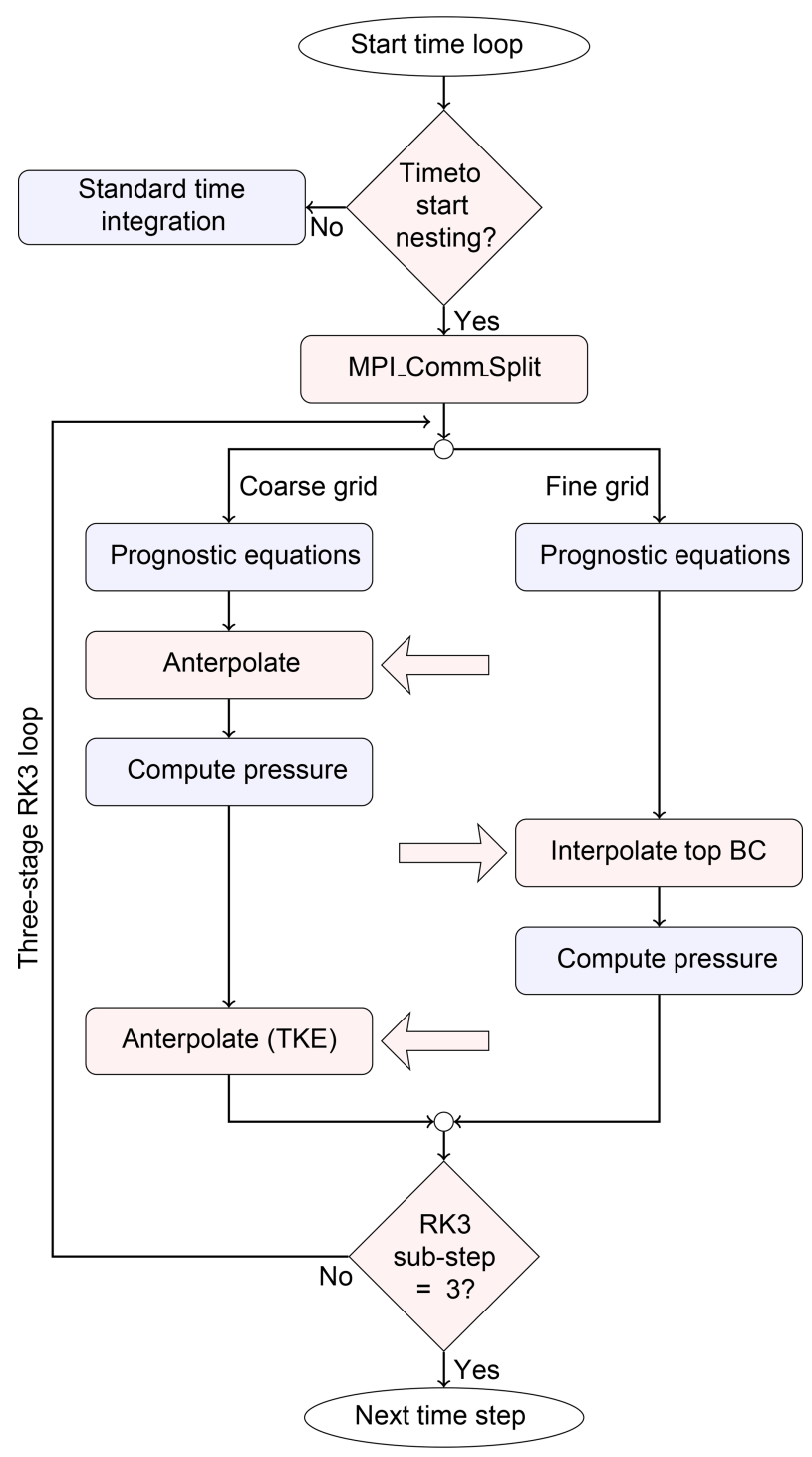

Figure 2. A flowchart of the two-way interaction algorithm. The new routines needed for the vertical nesting are highlighted in red and the standard routines are highlighted in blue. An arrow pointing to the left indicates transfer of data from FG to $\mathrm{CG}$, and vice versa.

$$
\begin{aligned}
\eta_{-}^{m} & =\frac{1}{2} H_{m}\left(H_{m}-1\right)+\alpha, \\
\eta_{0}^{m} & =\left(1-H_{m}^{2}\right)-2 \alpha, \\
\eta_{+}^{m} & =\frac{1}{2} H_{m}\left(H_{m}+1\right)+\alpha,
\end{aligned}
$$

with the weights $H_{m}$ expressed in function of the inverse nesting ratio,

$$
H_{m}=\frac{1}{2}\left((2 m-1) f_{x}-1\right),
$$

and the coefficient $\alpha$ is chosen such that the conservation condition of Kurihara et al. (1979) is satisfied:

$\alpha=\frac{1}{24}\left(f_{x}^{2}-1\right)$.

It can be observed that the sum of the $\eta$ s equals 1 .

\subsubsection{Anterpolation}

The anterpolation of the prognostic quantities is performed by an averaging procedure according to Clark and Hall (1991). The anterpolation equations for the velocities read as

$$
\begin{aligned}
U_{I, J, K} & =<u>_{j, k}=\sum_{j, k \in \mathcal{C}_{\mathcal{I} \mathcal{J K}}} u_{i^{*}, j, k} f_{y} f_{z}, \\
V_{I, J, K} & =<v>_{i, k}=\sum_{i, k \in \mathcal{C}_{\mathcal{I}} \mathcal{J K}} v_{i, j^{*}, k} f_{x} f_{z}, \\
W_{I, J, K} & =<w>_{i, j}=\sum_{i, j \in \mathcal{C}_{\mathcal{I} \mathcal{J K}}} w_{i, j, k^{*}} f_{x} f_{y} .
\end{aligned}
$$

For the scalars it is

$$
\Phi_{I, J, K}=[\phi]_{i, j, k}=\sum_{i, j, k \in \mathcal{C}_{\mathcal{I} \mathcal{J K}}} \phi_{i, j, k} f_{x} f_{y} f_{z} .
$$

Here the lower case indices only count over the fine grid cells that belong to that particular coarse grid cell. For each $(I, J, K)$ tuple of a parent $\mathrm{CG}$ cell, there exists a set $\mathcal{C}_{\mathcal{I} \mathcal{J K}}$ containing the $(i, j, k)$ tuples of its corresponding children FG cells. To ensure that the nested PALM knows at all times which fine grid cells and coarse grid cells correspond, we compute this mapping for the FG and CG indices before starting the simulation, and we store it in the memory of the parallel processing element. In the Arakawa $\mathrm{C}$ grid discretization that PALM uses, the scalars are defined as the spatial average over the whole grid cell, and therefore it is required that the CG scalar is the average of the corresponding FG scalars in (Eq. 15). However, the velocities are defined at the faces of the cells in the corresponding dimension. Therefore in (Eq. 14) the CG velocity components are computed as the average over the FG values at the FG cells that correspond to the face of the CG cell, expressed by $i^{*}, j^{*}, k^{*}$ respectively.

However, the TKE in the CG differs from the FG value, due to the different resolution of grids. In the FG the SGS motions are weaker because the turbulence is better resolved. Therefore, TKE is anterpolated such that the sum of resolved kinetic energy and TKE (SGS kinetic energy) is preserved, by maintaining the Germano identity (Germano et al., 1991):

$E=[e]+\frac{1}{2} \sum_{n=1}^{3}\left(\left[u_{n} u_{n}\right]-\left[u_{n}\right]\left[u_{n}\right]\right)$.

Here the straight brackets are the spatial average over the coarse grid cell $\left(f_{x} f_{y} f_{z} \times \sum_{i, j, k \in \mathcal{C}_{I J K}}\right)$, and the $n$ index runs 


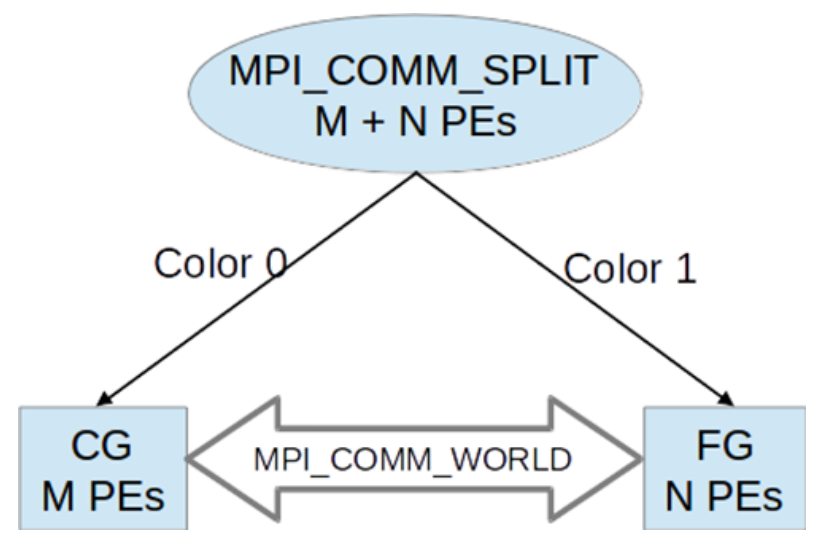

Figure 3. Schematic of the MPI processor grouping. The data exchange between the two groups is performed via the global communicator. $M$ and $N$ are the number of processors for CG and FG respectively.

over the three spatial dimensions. In other words, to obtain the CG TKE from the average FG TKE, we add the variance of the FG velocity components over the FG cells comprising the $\mathrm{CG}$ cell. Therefore CG TKE is always larger than FG TKE.

\subsection{Parallel inter-grid communication}

MPI is the most widely used large-scale parallelization library. The atmosphere-ocean coupling in PALM has been implemented following MPI-1 standards (Esau, 2014; Maronga et al., 2015). We follow a similar approach for the MPI communications and have adopted MPI-1 standards for our nesting implementation. Concurrent execution of the two grids is achieved with the MPI_COMM_SPLIT procedure. The total available processors are split into two groups, denoted by color 0 or 1 for $\mathrm{CG}$ and FG respectively; see Fig. 3. The data between the processors of the same group are exchanged via the local communicator created during the splitting process, whereas the data between the two groups are exchanged via the global communicator MPI_COMM_WORLD.

Based on the nesting ratio and the processor topology of the FG and the CG group, a mapping list is created and stored. Given the local PE's 2-D processor co-ordinate, the list will identify the PEs in the remote group to/from which data need to be sent/received; the actual communication then takes place via the global communicator. There are three types of communication in the nesting scheme:

i. Initialization of the FG. (Send data from coarse grid to fine grid.) This is performed only once.

ii. Boundary condition for the FG top face. (Send data from coarse grid to fine grid.)

iii. Anterpolation. (Send data from fine grid to coarse grid.)
The exchange of arrays via MPI_SENDRECV routines is computationally expensive. Therefore, the size of the arrays communicated is minimized by performing the anterpolation operation in the FG PEs and storing the values in a temporary 3-D array that is later sent via the global communicator to the appropriate CG PE. This approach is more efficient than performing the anterpolation operation on the $\mathrm{CG}$ which has fewer PEs and needs communication of larger arrays from the FG. Furthermore, the array data that need to be communicated during the anterpolation operation and for setting the FG boundary condition are not contiguous in memory. The communication performance is enhanced by creating an MPI-derived data type that ensures that the data are sent contiguously. Within the RK3 sub-steps, when one grid executes the pressure solver, the other grid has to wait, leading to more computational time at every sub-step. However, the waiting time can be minimized by effective load balancing; i.e., the number of grid points per PE in the CG should be kept lower than in the FG. The reduction in workload per CG PE is achieved with a few additional cores. The reduction in computational time per step in the CG means the waiting time on the FG PE is also reduced.

\section{Results and discussion}

\subsection{Simulation setup for the nesting validation test}

To evaluate the accuracy of the two-way nesting algorithm, we set up a convective boundary layer simulation. Two overlapping grids with a nesting ratio of 5 in the lateral and vertical direction are employed. The simulation parameters are listed in Table 2. A stand-alone reference simulation with the same resolution as the coarse grid (SA-C) and another reference with the same resolution as the fine grid (SA-F) are performed for comparison. The grid configuration and the computational resources used are listed in Table 3. The simulations were performed in a local computing cluster; each compute node has $64 \mathrm{~GB}$ of main memory and a $2.8 \mathrm{GHz}$ Ivy Bridge processor with 20 cores. The simulation domain has periodic boundary conditions in the lateral direction. The Dirichlet boundary condition is applied for velocity at the top and bottom boundaries, the vertical velocity component is set to zero and the horizontal components are set to geostrophic wind. At the top and bottom boundaries, the pressure and humidity are set to a zero gradient Neumann condition. The potential temperature is set to a Neumann condition at the bottom, and the gradient is determined by MOST based on the prescribed surface heat flux and roughness length. The gradient of the initial profile is maintained at the top boundary. In PALM, $u_{\mathrm{g}}$ and $v_{\mathrm{g}}$ represents the $u$ and $v$ component of the geostrophic wind at the surface. The $u$ and $v$ initial profiles are set to be constant, equal to the value of the geostrophic wind component in the domain, and the vertical velocity is initialized to zero in the domain. The potential 
temperature is initialized to a constant value of $300 \mathrm{~K}$ up to $800 \mathrm{~m}$, and above $800 \mathrm{~m}$ a lapse rate of $1 \mathrm{~K}(100 \mathrm{~m})^{-1}$ is prescribed. The humidity profile is initialized to a constant value of $0.005 \mathrm{~kg} \mathrm{~kg}^{-1}$. The simulation is driven by prescribing a surface heat flux of $0.1 \mathrm{~K} \mathrm{~m} \mathrm{~s}^{-1}$ and a surface humidity flux of $4 \times 10^{-4} \mathrm{~kg} \mathrm{~kg}^{-1} \mathrm{~m} \mathrm{~s}^{-1}$. The domain is more than 4 times larger in the horizontal than the initial boundary layer height.

\subsection{Analysis of the simulations}

In a two-way nesting it is important that the flow structures are propagated from the FG to $\mathrm{CG}$ and vice versa, without any distortion. In Fig. 4, the contours in the $\mathrm{CG}$ region overlapping the FG have similar structures as the FG. The higher resolution in the FG enables more detailed contours, whereas the anterpolated $\mathrm{CG}$ contours are smoother. Furthermore, in the $\mathrm{CG}$ region beyond the overlapping region, no distortion of the contours is observed, indicating that the anterpolation does not introduce sharp gradients in the CG.

Vertical profiles are used for quantitative comparison of the nested and the reference simulations. The turbulent fluctuations (e.g., $\left.\theta^{\prime \prime}, w^{\prime \prime}\right)$ are defined as the spatial deviations from the instantaneous horizontal average. The turbulent fluxes (e.g., $<\overline{w^{\prime \prime} \theta^{\prime \prime}>},<\overline{u^{\prime \prime} u^{\prime \prime}}>$ ) are obtained using the spatial covariance and are then horizontally averaged. All the horizontally averaged profiles (e.g., $\langle\theta\rangle,\left\langle w^{\prime \prime} \theta^{\prime \prime}\right\rangle$ ) are also averaged over time, but we omit the conventional overline notation for readability. The convective velocity scale $\left(w_{*}\right)$ and temperature scale $\left(\theta_{*}\right)$ obtained from SA-F are used to normalize the profiles. The convective velocity is calculated as $w_{*}=\left(g \theta_{0}^{-1} H_{\mathrm{S}} z_{i}\right)^{1 / 3}$, where $g$ is the gravitational acceleration, $\theta_{0}$ is the surface temperature and $z_{i}$ is the boundary layer height in the simulation. The convective temperature scale is calculated as $\theta_{*}=H_{\mathrm{s}} w_{*}^{-1}$. In Fig. 5a and c, the vertical profiles of difference between the potential temperature $(\langle\theta\rangle)$ and its surface value normalized by the convective temperature scale are plotted. Since the FG profiles are superior to the $\mathrm{CG}$ in the overlapping region, the anterpolated $C G$ values are not plotted. In Fig. 5a, there is no visible difference between the stand-alone and the nested simulations. However, in the region closer to the surface, plotted in Fig. 5c, a better agreement between the SA-F and FG is observed. The potential temperature variance $\left.\left(<\theta^{\prime \prime} \theta^{\prime \prime}\right\rangle\right)$ normalized by the square of the temperature scale $\left(\theta_{*}^{2}\right)$ is shown in Fig. $5 b$ and d. Here too FG provides better accuracy close to the surface.

In the vertical heat flux $\left(<w^{\prime \prime} \theta^{\prime \prime}>\right)$ profiles in Fig. 6 , the FG has good agreement with the SA-F in the surface layer for the resolved, SGS and the total flux profiles. In the CG regions above the nested grid height, a good agreement with the SA-C is found as well. The improvement due to the twoway nesting is seen in Fig. 6d and e, where the effects of low grid resolution of the SA-C in resolved and SGS fluxes are evident. However, no grid-dependent difference in the profile is observed in the total flux.
The resolved variances of $u, v$ and $w$ normalized by the square of the convective velocity $\left(w_{*}^{2}\right)$ are plotted in Fig. 7. The FG $v$ and $w$ FG profiles have a better agreement with the SA-F than the $u$ variance. The $u$ and $v$ variances in Fig. $7 \mathrm{~d}$ and e lie between SA-C and SA-F, indicating that the resolved variances are improved compared to the SA-C but not sufficiently resolved to match SA-F. At the nesting height the variances deviate more from the SA-F and approach the CG values. Due to conservation of total kinetic energy across the nest boundary, more CG TKE is contained in the sub-grid scale. Consequently, the resolved $\mathrm{CG}$ variances could have an undershoot as compared to SA-F, resulting in an undershoot of the FG variances too at the nesting height. Above the nesting height, the variance of $u, v$ and $w$ in CG is similar to SA-C.

The resolved vertical velocity skewness in Fig. 8 shows good agreement between the FG and SA-F close to the surface. However, at the nesting height a small kink in the skewness is noticeable. Zhou et al. (2018) observe that the magnitude of the kink in the higher-order profiles can be minimized by increasing the depth of the sponge layer. Our simplified sponge layer approach appears to be unable to effectively minimize the kinks at the nesting height. The resolved skewness in CG is lower than SA-C, possibly due to larger SGS TKE in the CG, as seen in Fig. 8d. The SGS TKE in Fig. 8d shows an exact match between FG and SA-F close to the surface and only marginal difference at the nesting height. However, CG values are considerably different from the SA$\mathrm{C}$ values close to the surface due to the anterpolation maintaining the Germano identity for conservation of kinetic energy across the grids. In the coarse-resolution SA-C, near the surface, the SGS turbulence model appears to insufficiently model the SGS effects. Above the nesting height the $\mathrm{CG}$ is similar to SA-C.

The horizontal spectra of SGS turbulent kinetic energy and vertical velocity are plotted in Fig. 9 at two levels, one within the nested grid and one above the nested grid height. The FG TKE spectra in Fig. 9c perfectly overlap the SA-F spectra. The CG spectra have higher energy than the SA-C; this corresponds to the higher CG TKE values observed in Fig. 8c. As the limit of the grid resolution is reached at high wavenumber, the drop in the CG spectra is marginally shifted compared to SA-C. This improvement at high wavenumber is due to feedback from the FG. Similarly, the vertical velocity spectra in Fig. 8d show marginal improvement at high wavenumber for the $\mathrm{CG}$ with respect to SA-C. While the FG agrees with SA-C at high wavenumber and at the spectra peak, at low wavenumber FG follows the CG spectra. At the level above the nested grid, the CG spectra agree with SA-C for both TKE and the vertical velocity.

\subsection{Computational performance}

The computational resources used in the simulations discussed above are listed in Table 3 . The resources needed 
Table 2. Simulation parameters for the nesting validation test.

\begin{tabular}{ll}
\hline Simulation parameters & Value \\
\hline Domain size: & $4.0 \times 4.0 \times 1.65 \mathrm{~km}^{3}$ \\
Fine grid vertical extent: & $320 \mathrm{~m}$ \\
Kinematic surface heat flux: & $H_{\mathrm{s}}=0.1 \mathrm{~K} \mathrm{~m} \mathrm{~s}^{-1}$ \\
Kinematic surface humidity flux: & $\lambda E_{\mathrm{s}}=4 \times 10^{-4} \mathrm{~kg} \mathrm{~kg}^{-1} \mathrm{~m} \mathrm{~s}^{-1}$ \\
Geostrophic wind: & $u_{\mathrm{g}}=1 \mathrm{~m} \mathrm{~s}^{-1}, v_{\mathrm{g}}=0 \mathrm{~m} \mathrm{~s}^{-1}$ \\
Roughness length: & $0.1 \mathrm{~m}$ \\
Simulated time: & $10800 \mathrm{~s}$ \\
Spin-up time: & $9000 \mathrm{~s}$ \\
Averaging interval: & $1800 \mathrm{~s}$ \\
\hline
\end{tabular}

Table 3. Grid configuration of the nested and stand-alone reference domains.

\begin{tabular}{llrrrrr}
\hline Case & $\begin{array}{l}\text { No. of } \\
\text { grid points }\end{array}$ & $\begin{array}{r}(\mathrm{d} x, \mathrm{~d} y, \mathrm{~d} z) \\
\mathrm{m}\end{array}$ & $\begin{array}{r}\text { CPU } \\
\text { cores }\end{array}$ & $\begin{array}{r}\text { Core } \\
\text { hours }\end{array}$ & $\begin{array}{r}\text { Grid points } \\
\text { per core }\end{array}$ & $\begin{array}{r}\text { Time } \\
\text { steps }\end{array}$ \\
\hline Coarse grid (CG) & $200 \times 200 \times 80=3.2 \times 10^{6}$ & $20,20,20$ & 20 & 376 & $1.6 \times 10^{5}$ & 17136 \\
Fine grid (FG) & $1000 \times 1000 \times 80=80 \times 10^{6}$ & $4,4,4$ & 80 & 1503 & $1.0 \times 10^{6}$ & 17136 \\
\hline Total & & & 1879 & \\
\hline Stand-alone coarse (SA-C) & $200 \times 200 \times 80=3.2 \times 10^{6}$ & $20,20,20$ & 20 & 8 & $1.6 \times 10^{5}$ & 3226 \\
\hline Stand-alone fine (SA-F) & $1000 \times 1000 \times 400=400 \times 10^{6}$ & $4,4,4$ & 400 & 8234 & $1.0 \times 10^{6}$ & 18343 \\
\hline
\end{tabular}

Table 4. Number of grid points in nested and non-nested FG domain.

\begin{tabular}{lr}
\hline Case & No. of grid points \\
\hline Coarse grid & $840 \times 840 \times 288=0.20 \times 10^{9}$ \\
Fine grid & $4200 \times 4200 \times 360=6.35 \times 10^{9}$ \\
\hline Total & $6.55 \times 10^{9}$ \\
\hline Non-nested FG & $4200 \times 4200 \times 360=6.35 \times 10^{9}$ \\
\hline
\end{tabular}

by SA-C is only 8 core hours. While the nested simulations needed about 1879 core hours, the SA-F needed about 4 times more core hours than the nested simulation. As the resolution is increased from $20 \mathrm{~m}$ in SA-C to $4 \mathrm{~m}$ in SA-F, the number of time steps increased more than 5 times as higher resolution demands a smaller time step size. Though the number of time steps in FG is similar to SA-F, limiting the nested grid in the vertical direction has reduced the number of CPU cores needed, and higher resolution in the surface layer is achieved at a reduced computational cost.

Several factors influence the computational performance of an LES code. Some factors depend on the hardware; e.g., the number of grid points per PE depends on the memory available per node. On the other hand, the communication time for data exchange between the PEs depends on the topology of the domain decomposition. The best performance in terms of communication time in a stand-alone run is achieved when the number of sub-domains in the $x$ and $y$ directions is equal. In that case the number of ghost points at the lateral boundaries is optimally minimized. In a nested simulation, the load per PE, i.e., the number of grid points per $\mathrm{PE}$, in the two grids varies. As the speed of the model integration depends on the PE load, the load balancing between the fine and coarse grid has an effect on the computational performance of the nested simulation. Keeping these factors in mind, we designed the nested simulation domains listed in Table 4 for the purpose of assessing the computational performance, as the total number of processors is varied. To avoid load balancing bias in the scalability analysis, the ratio between the number of PEs for CG and FG is kept constant in all the five runs listed in Table 5. Keeping the processor ratio constant implies that the ratio between the number of grid points per PE in CG and FG is also held constant. Consequently, in this performance test, the FG has 1.25 times more grid points per PE than the CG in all the processor configurations tested. To compare the performance of the nested model against the non-nested version of PALM under equivalent work load, a grid with the same dimensions of the FG is set up. This non-nested grid also has the same load per PE and same number of cores as the FG. Such a non-nested setup is acceptable for comparison since the number of PEs in $\mathrm{CG}$ is negligible compared to the PE in FG in our setup (e.g., 14400 PEs in FG and only 576 PEs in CG). A pure standalone simulation with FG resolution throughout the boundary layer was not performed as it would need about $2.5 \times 10^{10}$ 

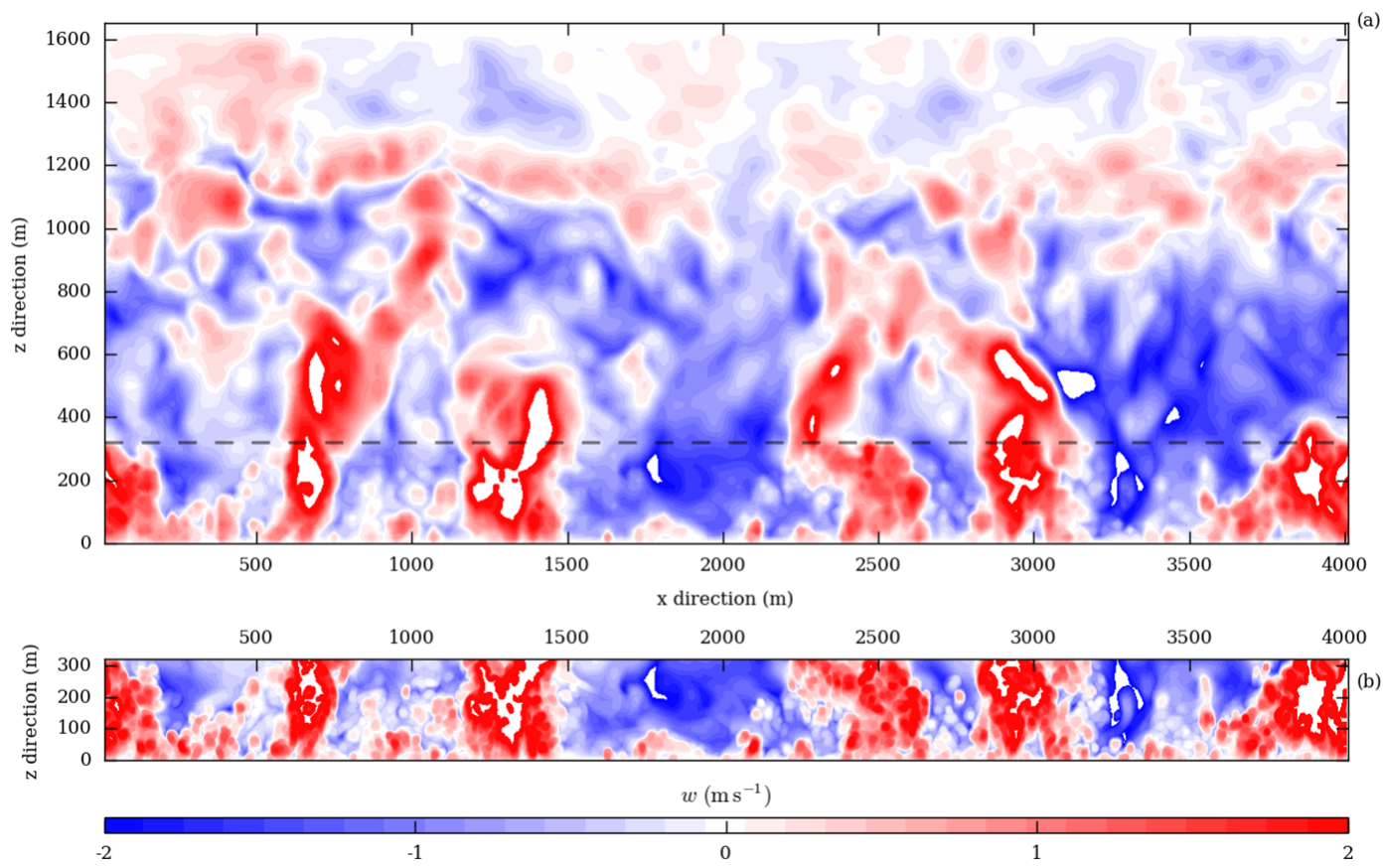

Figure 4. Instantaneous contours of vertical velocity, (a) CG and (b) FG, at the vertical $x-z$ cross section at the center of the domain after $10800 \mathrm{~s}$ of the simulation. The dashed line in (a) marks the top of the overlapping region. Flow structures in the FG, which are similar but more detailed than the CG, qualitatively indicate the improvement to the surface layer resolution with the two-way nesting.
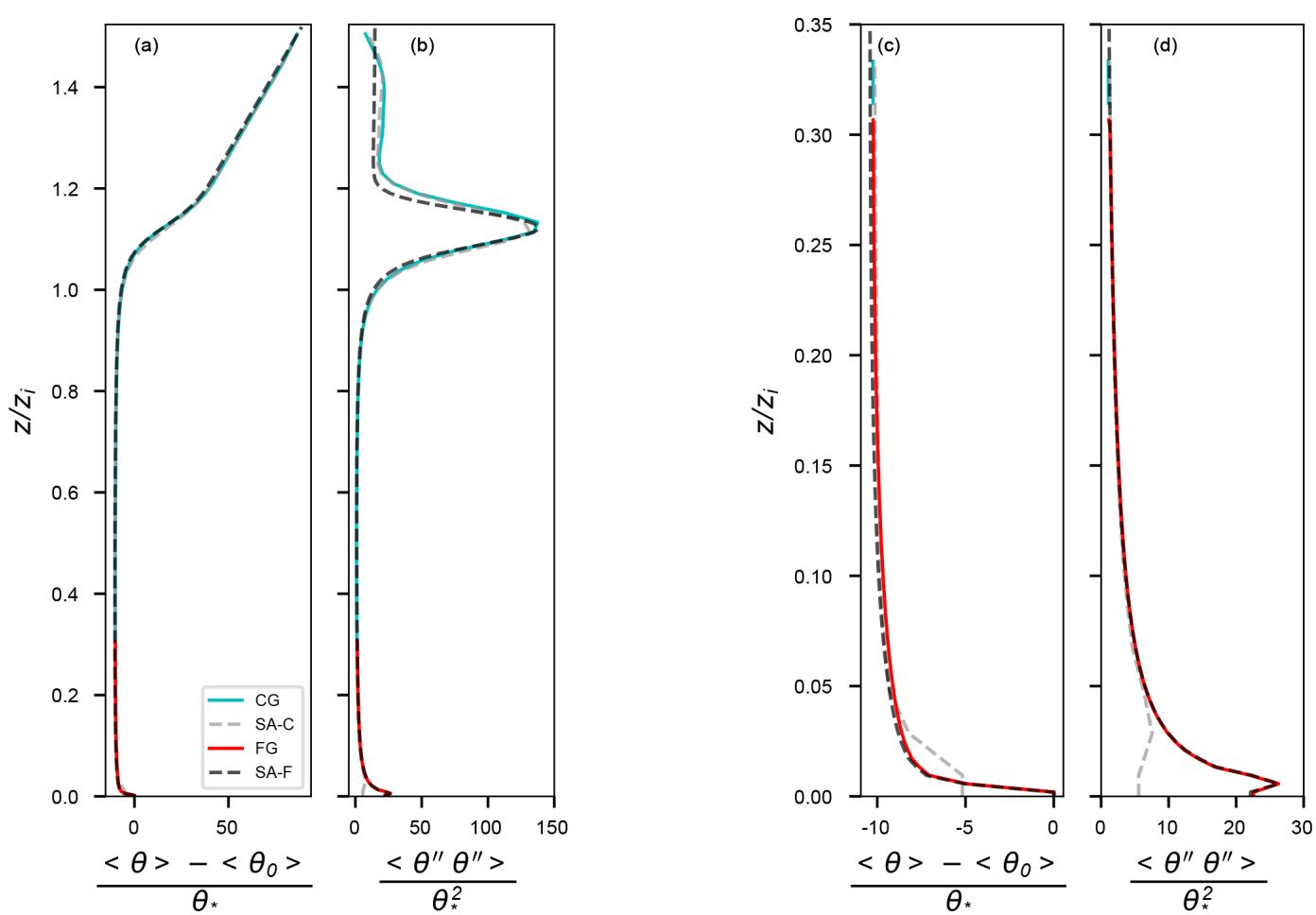

Figure 5. Vertical profile of horizontally averaged potential temperature normalized by the surface value (a, c) and variance of potential temperature normalized by $\theta_{*}^{2}(\mathbf{b}, \mathbf{d})$. The nested grid profiles agree well with the SA-F in the surface layer. The improvement of the two-way nesting, at the boundary layer height, is seen in the good agreement in the profiles of CG and SA-F in (b). 

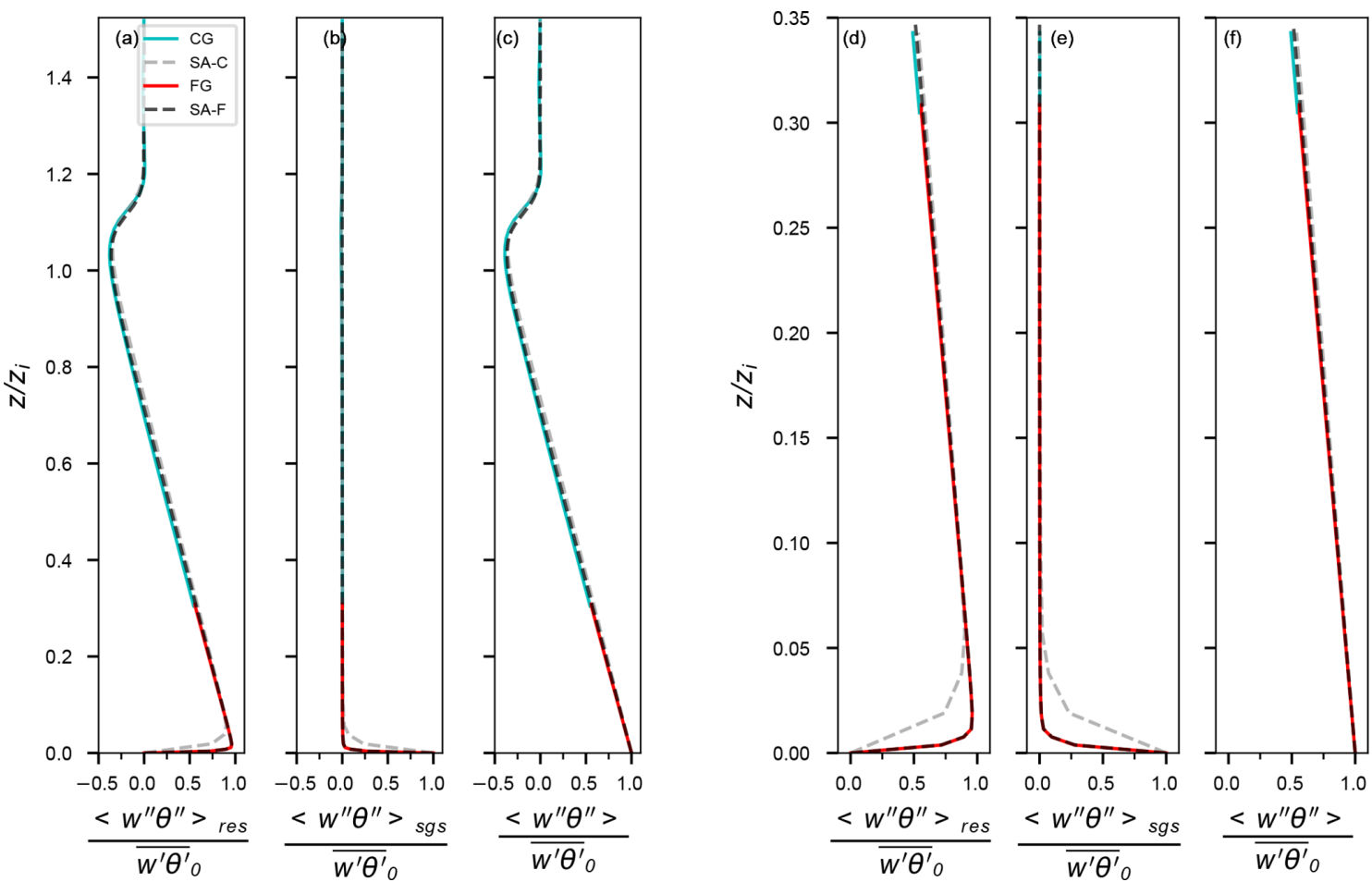

Figure 6. Vertical profile of horizontally averaged heat flux normalized by the surface heat flux - resolved (a, d), sub-grid (b, e) and total flux (c, f). The two-way nesting significantly improves the resolved and SGS fluxes in the surface layer.

Table 5. Grid configuration of the nested and non-nested FG domain.

\begin{tabular}{lrrrrr|rrr}
\hline & \multicolumn{7}{c|}{ Nested } & \multicolumn{3}{c}{ Non-nested FG } \\
\cline { 2 - 9 } Run & Total & CG & FG & $\begin{array}{r}\text { Avg. time } \\
\text { Per step (s) }\end{array}$ & $\begin{array}{r}\text { Efficiency } \\
(\%)\end{array}$ & $\begin{array}{r}\text { Total } \\
\text { PE }\end{array}$ & $\begin{array}{r}\text { Avg. time } \\
\text { per step (s) }\end{array}$ & $\begin{array}{r}\text { Efficiency } \\
(\%)\end{array}$ \\
\hline A & 1664 & 64 & 1600 & 44.0 & 100 & 1600 & 14.9 & 100 \\
B & 3744 & 144 & 3600 & 19.9 & 98 & 3600 & 6.7 & 99 \\
C & 7488 & 288 & 7200 & 10.3 & 95 & 7200 & 3.6 & 92 \\
D & 8736 & 336 & 8400 & 9.3 & 90 & 8400 & 3.4 & 84 \\
E & 14976 & 576 & 14400 & 5.6 & 87 & 14400 & 2.3 & 74 \\
\hline
\end{tabular}

grid points, and such a large domain was computationally not feasible. The performance is measured in terms of the time taken to simulate one time step. To increase the accuracy of this performance measurement, the simulation is integrated for 10 time steps, and the average of the time per step is plotted. The results presented in Fig. 10 show close to linear scaling for up to 14976 PEs in both nested and stand-alone runs. The difference in time per step between the nested and stand-alone runs can be interpreted as the additional computational time needed by the nesting algorithm. A jump in the time taken to compute one step is observed when more than 8192 PEs are used. This is a hardware-dependent increase in communication time as the nodes are grouped as "islands" on SuperMUC system at the Leibniz Supercomputing Centre. The communication within the nodes of the same island is faster than the communication across multiple islands. The strong scaling efficiency in Table 4 is calculated, keeping the run with the lowest number of PEs as the reference. As the number of grid points per PE is reduced from run A to $E$ as shown in Table 5, the nested runs show slightly better efficiency than the non-nested runs. The average time per step of the nested grid is 3 times higher than the non-nested setup for run A, but the factor decreases to about 2.5 for run $\mathrm{E}$. This improvement is possibly due to reduction in waiting time between the FG and CG as the number of grid points per PE decreases.

\subsection{Practical considerations}

In this paragraph we summarize some guidelines for using this nesting approach. In PALM, the user has the choice to 

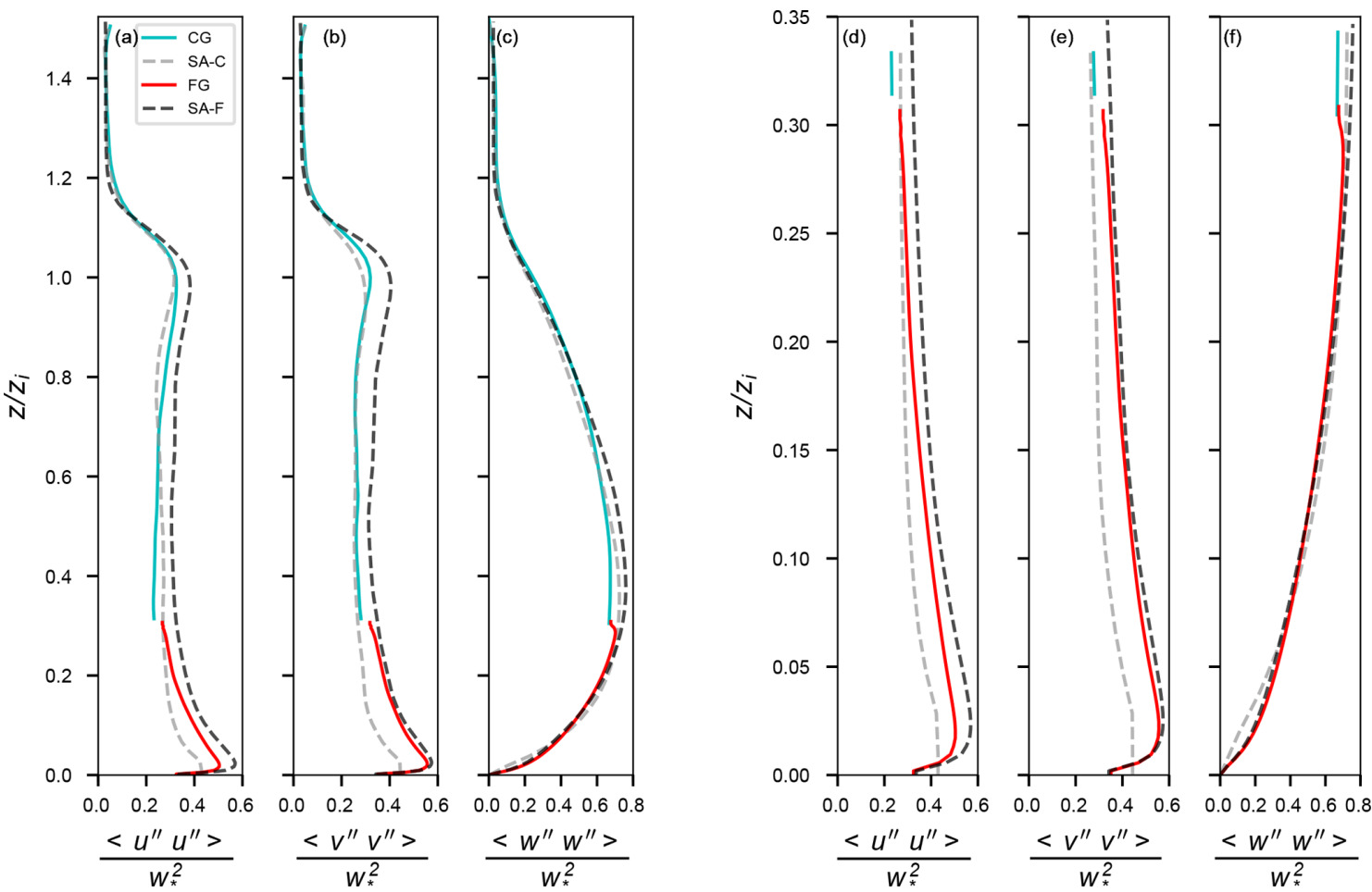

Figure 7. Vertical profile of horizontally averaged resolved variance of $u(\mathbf{a}, \mathbf{d}), v(\mathbf{b}, \mathbf{e})$, and $w(\mathbf{c}, \mathbf{f})$ normalized by $w_{*}^{2}$. The variance of $v$ and $w$ shows better agreement with the stand-alone reference in the surface layer.
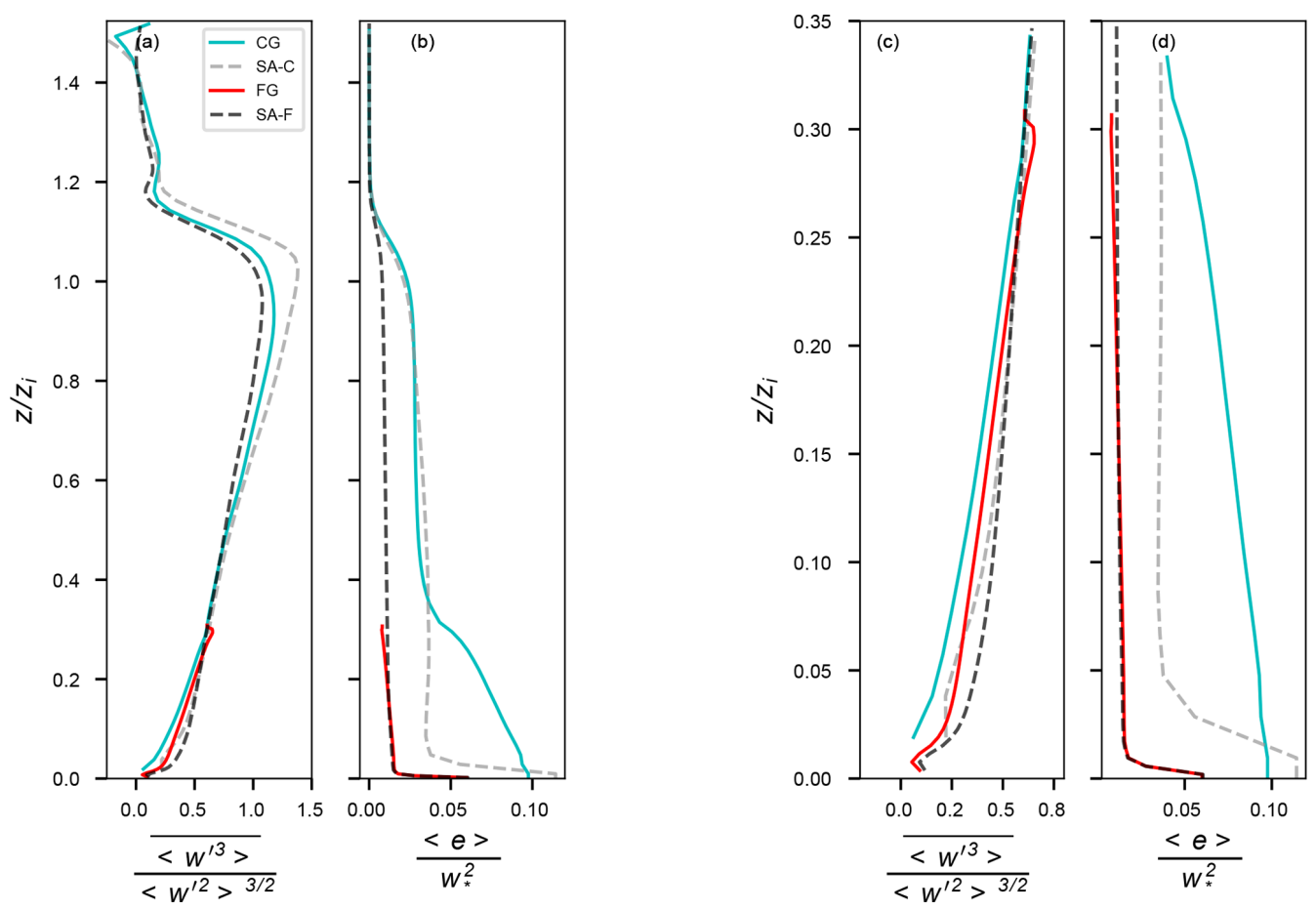

Figure 8. Vertical profile of horizontally averaged resolved vertical velocity skewness (a, c) and SGS turbulent kinetic energy $e$ (b, d) normalized by $w_{*}^{2}$. The SGS TKE in the CG is higher than SA-C as a result of anterpolation maintaining the Germano identity. 

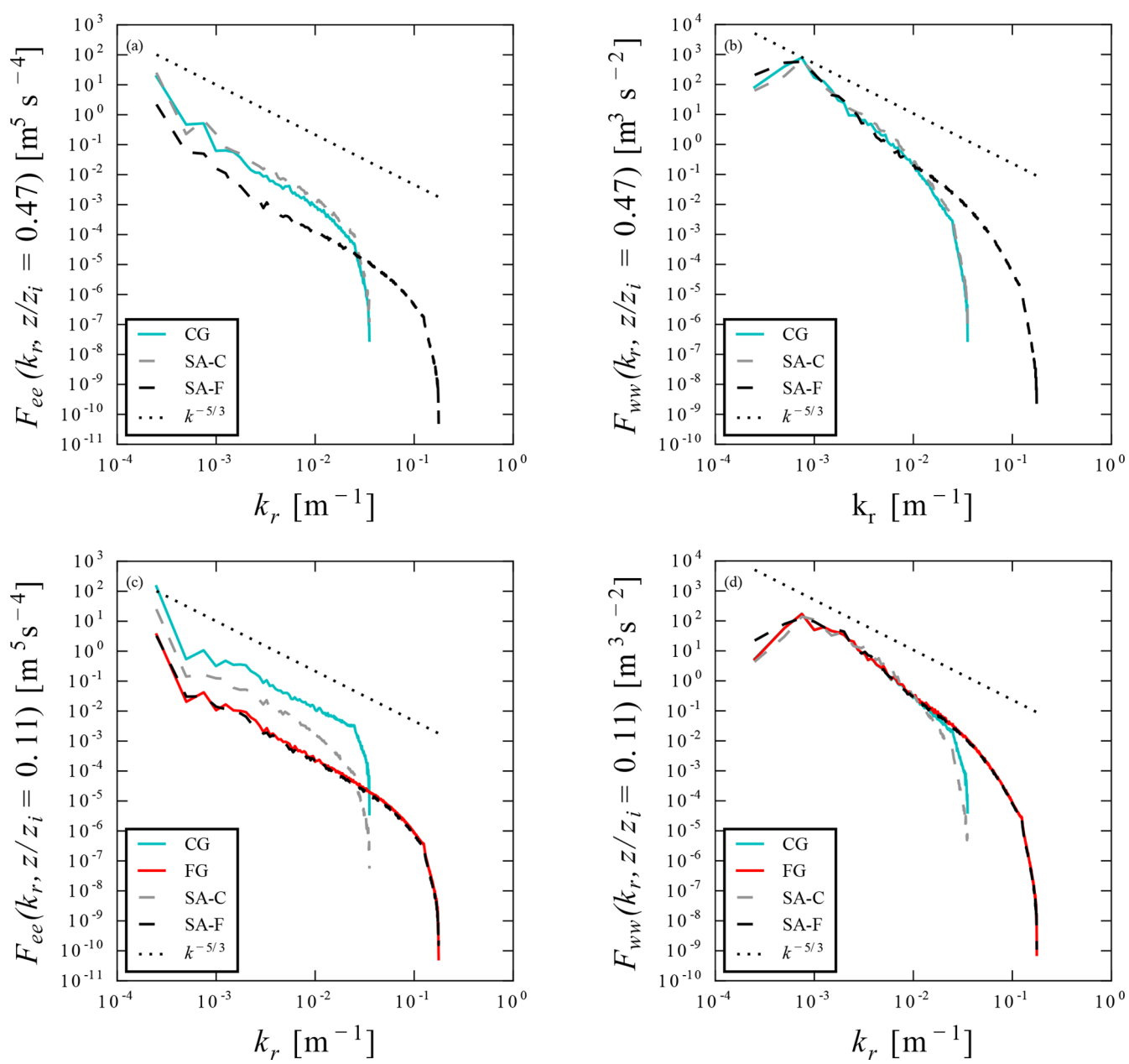

Figure 9. Spectra of SGS turbulent kinetic energy $(e)(\mathbf{a}, \mathbf{c})$ and vertical velocity $(w)(\mathbf{b}, \mathbf{d})$. At $z / z_{i}=0.47(\mathbf{a}, \mathbf{b})$ and at $z / z_{i}=0.11(\mathbf{c}, \mathbf{d})$, $k_{r}$ is the horizontal wavenumber.

select between Wicker-Skamarock (Wicker and Skamarock, 2002) and Piacsek-Williams (Piacsek and Williams, 1970) for the advection scheme. Similarly, for solving the Poisson equation for the pressure, the user can choose between the FFT or multi-grid-based solver. During the development and the validation of the two-way nesting, only the Wicker-Skamarock advection scheme and FFT-based pressure solvers were tested. The two-way nesting supports only periodic boundary conditions in the horizontal for both CG and FG, and therefore an FFT-based pressure solver is an appropriate choice. However, to be able to use multigrid solvers, e.g., in nonperiodic horizontal boundary conditions, modifications to the two-way nesting algorithm will be needed. The large-scale forcing feature in PALM is found to be compatible with the nesting algorithm without further modifications. Other features like canopy parameterization, radiation models and land surface models have not been tested.

Our implementation of the vertical nesting allows only integer nesting ratios in all directions. The height of the nested domain has a direct influence on the accuracy of the twoway nesting algorithm. Based on our trials (not shown) we recommend that the FG covers at least 12 grid levels of the CG. For better computational performance we recommend that the number of grid points per PE in the CG is kept at only $40 \%$ to $80 \%$ of the FG value. The reduced work load of the $\mathrm{CG}$ is expected to minimize the waiting time of the FG during the concurrent time advancement by the quicker CG pressure solver step. However, the actual improvement in performance will depend on the memory available, processor speed and the inter-node communication architecture of the computing cluster, and the optimal load balancing can only be found through trials. Furthermore, the choice of the domain size is often restricted by the topology of the processor decomposition. In a 2-D decomposition, the number of grid points along the $x$ direction should be an integer multiple of the number of PEs along $x$ and similarly for the $y$ direction. This condition has to be individually satisfied for the CG and the FG. Though our nesting technique makes resolving the surface layer resolution down to $0.5 \mathrm{~m}$ for a moderately large 


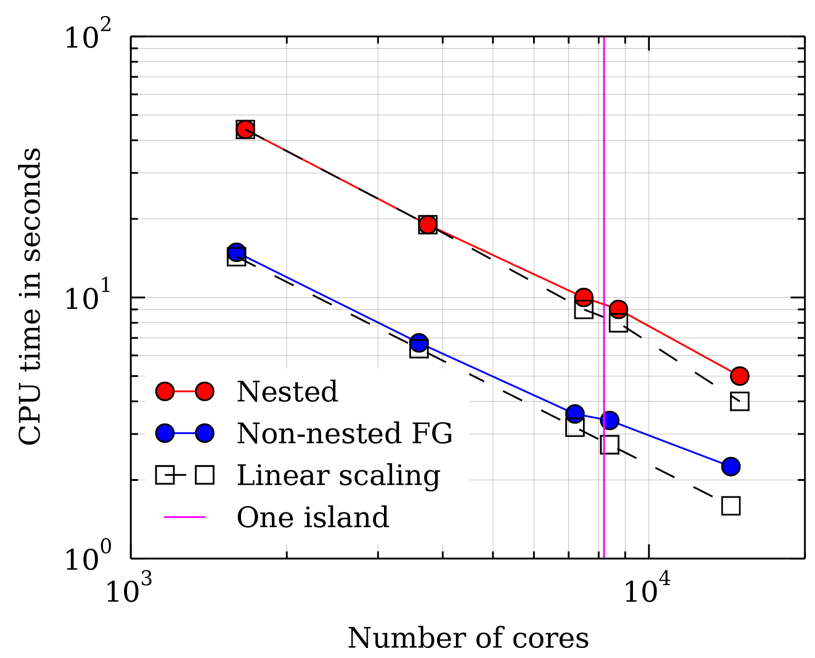

Figure 10. The nested simulations show close to linear scalability. A non-nested domain with same number of grid points as the FG is plotted to benchmark the scalability of the standard version of PALM on the same machine. The difference between the blue and the red line is approximately equal to the additional computational time needed by the nesting routines. The simulations were performed on SuperMUC at the Leibniz Supercomputing Centre. Each node has $32 \mathrm{~GB}$ of main memory and two Sandy Bridge processors with $2.7 \mathrm{GHz}$; each processor has eight cores (Anastopoulos et al., 2013).

domain computationally feasible, care should be taken to ensure the validity of such LES. In PALM, the height of the first grid point should be at the least twice greater than the local surface-roughness parameter. This technical restriction is common to all models that employ MOST and ensures the proper evaluation of the logarithm needed in the calculation of $u^{*}$. Furthermore, Basu and Lacser (2017) recently recommended that MOST boundary conditions should be adapted for very high resolution LES, where the first grid point is smaller than 2-5 times the height of the roughness elements.

\section{Summary}

We presented a two-way grid nesting technique that enables high-resolution LES of the surface layer. In our concurrently parallel algorithm, the two grids with different resolution overlap in the region close to the surface. The grids are coupled, i.e the interpolation of the boundary conditions and the feedback to the parent grid are performed, at every sub-step of the Runge-Kutta time integration. The anterpolation of the TKE involves the Germano identity to ensure the conservation of total kinetic energy. The exchange of data between the two grids is achieved by MPI communication routines, and the communication is optimized by derived data types. Results of the convective boundary layer simulation show that grid nesting improves the vertical profiles of variance and the fluxes in the surface layer. In particular, the profiles of the vertical temperature flux are improved. The current vertical nesting only works with periodic boundary conditions and with the same horizontal extent in both the domains. The nested simulation needs 4 times less computational time than a full high-resolution simulation for comparable accuracy in the surface layer. The scalability of the algorithm on up to 14976 CPUs is demonstrated.

Code availability. The Parallelized Large-eddy simulation Model (PALM) is developed and maintained by the PALM group, Institute of Meteorology and Climatology, Leibniz University Hannover (Raasch and Schröter, 2001; Maronga et al., 2015). The code is distributed under the GNU General Public License. The code (revision 2712) is available at https://palm.muk.uni-hannover.de/trac/ browser/palm?rev=2712 (last access: 24 June 2019).

Author contributions. SH was the main developer of the model code, with FDR as side developer, SR supporting the code development and MM, SR and FDR supervising the development. The experiment was designed by SH, FDR, SR and MM and carried out by $\mathrm{SH}$, who also performed the validation. Visualization was done by $\mathrm{SH}$ and the original draft written by $\mathrm{SH}$ and FDR, with review and editing by SR and MM. MM was responsible for funding acquisition and administration.

Acknowledgements. This work was conducted within the Helmholtz Young Investigators Group "Capturing all relevant scales of biosphere-atmosphere exchange - the enigmatic energy balance closure problem", which is funded by the Helmholtz Association through the President's Initiative and Networking Fund and by KIT. Computer resources for this project have been provided by the Leibniz Supercomputing Centre under grant pr48la. We thank Gerald Steinfeld for sharing his original notes and code of a preliminary nesting method in PALM. We also thank Matthias Sühring and Farah Kanani-Sühring of the PALM group for their help in standardizing and porting the code, and we thank Michael Manhart for fruitful discussions.

Financial support. This research has been supported by the Helmholtz Association (grant no. VH-NG-843).

The article processing charges for this open-access publication were covered by a Research Centre of the Helmholtz Association.

Review statement. This paper was edited by Christoph Knote and reviewed by Lucas Harris and two anonymous referees. 


\section{References}

Anastopoulos, N., Nikunen, P., and Weinberg, V.: Best Practice Guide - SuperMUC v1.0. PRACE - Partnership for Advanced Computing in Europe 2013, available at: http://www.prace-ri.eu/ best-practice-guide-supermuc-html (last access: 24 June 2019), 2013.

Basu, S. and Lacser, A.: A Cautionary Note on the Use of Monin-Obukhov Similarity Theory in Very High-Resolution Large-Eddy Simulations, Bound.-Lay. Meteorol., 163, 351-355, https://doi.org/10.1007/s10546-016-0225-y, 2017.

Boersma, B. J., Kooper, M. N., Nieuwstadt, F. T. M., and Wesseling, P.: Local grid refinement in large-eddy simulations, J. Eng. Math., 32, 161-175, https://doi.org/10.1023/A:1004283921077, 1997.

Clark, T. L. and Farley, R. D.: Severe downslope windstorm calculations in two and three spatial dimensions using anelastic interactive grid nesting: A possible mechanism for gustiness, J. Atmos. Sci., 41, 329-350, https://doi.org/10.1175/15200469(1984)041<0329:SDWCIT>2.0.CO;2, 1984.

Clark, T. L. and Hall, W. D.: Multi-domain simulations of the time dependent Navier Stokes equation: Benchmark error analyses of nesting procedures, J. Comput. Phys., 92, 456-481, https://doi.org/10.1016/0021-9991(91)90218-A, 1991.

Daniels, M. H., Lundquist, K. A., Mirocha, J. D., Wiersema, D. J., and Chow, F. K.: A New Vertical Grid Nesting Capability in the Weather Research and Forecasting (WRF) Model, Mon. Weather Rev., 144, 3725-3747, https://doi.org/10.1175/mwr-d16-0049.1, 2016.

Deardorff, J. W.: Three-dimensional numerical study of the height and the mean structure of a heated planetary boundary layer, Bound.-Lay. Meteorol., 7, 81-106, https://doi.org/10.1007/BF00224974, 1974.

Deardorff, J. W.: Stratocumulus-capped mixed layers derived from a three-dimensional model, Bound.-Lay. Meteorol., 18, 495-527, https://doi.org/10.1007/BF00119502, 1980.

Debreu, L., Marchesiello, P., Penven, P., and Cambon, L.: Twoway nesting in split-explicit ocean models: Algorithms, implementation and validation, Ocean Model., 49-50, 1-21, https://doi.org/10.1016/j.ocemod.2012.03.003, 2012.

Esau, I.: Indirect air-sea interactions simulated with a coupled turbulence-resolving model, Ocean Dynam., 64, 689-705, https://doi.org/10.1007/s10236-014-0712-y, 2014.

Flores, F., Garreaud, R., and Muñoz, R. C.: CFD simulations of turbulent buoyant atmospheric flows over complex geometry: Solver development in OpenFOAM, Comput. Fluids, 82, 1-13, https://doi.org/10.1016/j.compfluid.2013.04.029, 2013.

Germano, M., Piomelli, U., Moin, P., and Cabot, W. H.: A dynamic subgrid scale eddy viscosity model, Phys. Fluid A, 3, 1760-1765, https://doi.org/10.1063/1.857955, 1991.

Harris, L. M. and Durran, D. R.: An Idealized Comparison of OneWay and Two-Way Grid Nesting, Mon. Weather Rev., 138, 2174 2187, https://doi.org/10.1175/2010mwr3080.1, 2010.

Kato, C., Kaiho, M., and Manabe, A.: An Overset Finite-Element Large-Eddy Simulation Method With Applications to Turbomachinery and Aeroacoustics, J. Appl. Mech., 70, 32-43, https://doi.org/10.1115/1.1530637, 2003.

Khanna, S. and Brasseur, J. G.: Three-Dimensional Buoyancy- and Shear-Induced Local Structure of the Atmospheric Boundary Layer, J. Atmos.
Sci., $\quad 55, \quad 710-743, \quad$ https://doi.org/10.1175/1520 0469(1998)055<0710:tdbasi>2.0.co;2, 1998.

Kravchenko, A., Moin, P., and Moser, R.: Zonal Embedded Grids for Numerical Simulations of Wall-Bounded Turbulent Flows, J. Comput. Phys., 127, 412-423, https://doi.org/10.1006/jcph.1996.0184, 1996.

Kröniger, K., De Roo, F., Brugger, P., Huq, S., Banerjee, T., Zinsser, J., Rotenberg, E., Yakir, D., Rohatyn, S., and Mauder, M.: Effect of Secondary Circulations on the Surface-Atmosphere Exchange of Energy at an Isolated Semi-arid Forest, Bound.-Lay. Meteorol., 169, 209-232, https://doi.org/10.1007/s10546-018-0370-6, 2018.

Kurihara, Y., Tripoli, G. J., and Bender, M. A.: Design of a Movable Nested-Mesh Primitive Equation Model, Mon. Weather Rev., 107, 239-249, https://doi.org/10.1175/15200493(1979)107<0239:doamnm>2.0.co;2, 1979.

Manhart, M.: A zonal grid algorithm for DNS of turbulent boundary layers, Comput. Fluids, 33, 435-461, https://doi.org/10.1016/S0045-7930(03)00061-6, 2004.

Maronga, B., Gryschka, M., Heinze, R., Hoffmann, F., KananiSühring, F., Keck, M., Ketelsen, K., Letzel, M. O., Sühring, M., and Raasch, S.: The Parallelized Large-Eddy Simulation Model (PALM) version 4.0 for atmospheric and oceanic flows: model formulation, recent developments, and future perspectives, Geosci. Model Dev., 8, 2515-2551, https://doi.org/10.5194/gmd8-2515-2015, 2015.

Moeng, C.-H. and Wyngaard, J. C.: Spectral analysis of large-eddy simulations of the convective boundary layer, J. Atmos. Sci., 45, 3573-3587, https://doi.org/10.1175/15200469(1988)045<3573:SAOLES>2.0.CO;2, 1988.

Moeng, C.-H., Dudhia, J., Klemp, J., and Sullivan, P.: Examining Two-Way Grid Nesting for Large Eddy Simulation of the PBL Using the WRF Model, Mon. Weather Rev., 135, 2295-2311, https://doi.org/10.1175/MWR3406.1, 2007.

Nakahashi, K., Togashi, F., and Sharov, D.: Intergrid-Boundary Definition Method for Overset Unstructured Grid Approach, AIAA J., 38, 2077-2084, https://doi.org/10.2514/2.869, 2000.

Patton, E. G., Sullivan, P. P., Shaw, R. H., Finnigan, J. J., and Weil, J. C.: Atmospheric Stability Influences on Coupled Boundary Layer and Canopy Turbulence, J. Atmos. Sci., 73, 1621-1647, https://doi.org/10.1175/jas-d-15-0068.1, 2016.

Piacsek, S. A. and Williams, G. P.: Conservation properties of convection difference schemes, J. Comput. Phys., 198, 500-616, https://doi.org/10.1016/0021-9991(70)90038-0, 1970.

Raasch, S. and Schröter, M.: PALM - A large-eddy simulation model performing on massively parallel computers, Meteorol Z., 10, 363-372, https://doi.org/10.1127/0941-2948/2001/00100363, 2001.

Reynolds, W. C.: The potential and limitations of direct and large eddy simulations, in: Whither Turbulence? Turbulence at the Crossroads, edited by: Lumley, J. L., Springer Berlin Heidelberg, Berlin, Heidelberg, 313-343, https://doi.org/10.1007/3540-52535-1_52, 1990.

Saiki, E. M., Moeng, C.-H., and Sullivan, P. P.: Large-eddy simulation of the stably stratified planetary boundary layer, Bound.-Lay. Meteorol., 95, 1-30, https://doi.org/10.1023/A:1002428223156, 2000.

Schmidt, H. and Schumann, U.: Coherent structure of the convective boundary layer derived from large- 
eddy simulations, J. Fluid. Mech., 200, 511-562, https://doi.org/10.1017/S0022112089000753, 1989.

Skamarock, W., Klemp, J., Dudhia, J., Gill, D., Barker, D., Wang, W., Huang, X.-Y., and Duda, M.: A Description of the Advanced Research WRF Version 3, https://doi.org/10.5065/d68s4mvh, 2008.

Sullivan, P. P., McWilliams, J. C., and Moeng, C.-H.: A grid nesting method for large-eddy simulation of planetary boundary layer flows, Bound.-Lay. Meteorol., 80, 167-202, https://doi.org/10.1007/BF00119016, 1996.

van Hooft, J. A., Popinet, S., van Heerwaarden, C. C., van der Linden, S. J. A., de Roode, S. R., and van de Wiel, B. J. H.: Towards Adaptive Grids for Atmospheric BoundaryLayer Simulations, Bound.-Lay. Meteorol., 167, 421-443, https://doi.org/10.1007/s10546-018-0335-9, 2018.
Wang, G., Duchaine, F., Papadogiannis, D., Duran, I., Moreau, S., and Gicquel, L. Y.: An overset grid method for large eddy simulation of turbomachinery stages, J. Comput. Phys., 274, 333-355, https://doi.org/10.1016/j.jcp.2014.06.006, 2014.

Wicker, L. J. and Skamarock, W. C.: Time-splitting methods for elastic models using forward time schemes, Mon Weather Rev., 130, 2008-2097, https://doi.org/10.1175/15200493(2002)130<2088:TSMFEM>2.0.CO;2, 2002.

Williamson, J. H.: Low-storage Runge-Kutta schemes, J. Comput. Phys., 35, 48-56, https://doi.org/10.1016/0021-9991(80)900339, 1980.

Zhou, B., Xue, M., and Zhu, K.: A Grid-Refinement-Based Approach for Modeling the Convective Boundary Layer in the Gray Zone: Algorithm Implementation and Testing, J. Atmos. Sci., 75, 1143-1161, https://doi.org/10.1175/jas-d-17-0346.1, 2018. 